\section{Yield, Composition, and Antioxidant Capacity of Ground Cumin Seed Oil Fractions Obtained at Different Time Points during the Hydrodistillation}

\author{
Valtcho D. Zheljazkov ${ }^{1}$ and Santosh Shiwakoti \\ Oregon State University, Columbia Basin Agricultural Research Center, \\ 48037 Tubbs Ranch Road, Pendleton, OR 97801
}

\author{
Tess Astatkie \\ Faculty of Agriculture, Dalhousie University, 50 Pictou Road, P.O. Box 550, \\ Truro, NS B2N 5E3, Canada

\begin{abstract}
Ivan Salamon, Daniela Grul'ová, and Silvia Mudrencekova
Department of Ecology, Faculty of Humanities and Natural Sciences, Presov University, 01, 17th November Sreet, 08116 Presov, Slovak Republic
\end{abstract}

\section{Vicki Schlegel \\ Department of Food Science and Technology, University of Nebraska- Lincoln, 327 Food Technology Complex, Lincoln, NE 68583 \\ Additional index words. Cuminum cyminum, ORAC, cumin aldehyde, $\beta$-pinene, para-cymene, $\gamma$-terpinene}

\begin{abstract}
Cumin (Cuminum cyminum L.) is an important essential oil (EO), medicinal, and spice plant from family Apiaceae. Cumin seed EO has wide applications in the food, liquor, pharmaceutical, and aromatherapy industries, and is extracted via steam or hydrodistillation of either whole or ground seed. The hypothesis of this study was that by capturing oil eluted at different timeframes during the hydrodistillation process (HDP), we could obtain oils of differential composition and bioactivity. The objective was to evaluate the EO fractions captured at different timeframes of the HDP. In this study, we collected nine different EO fractions following nine hydrodistillation time (HDT) frames: 0-2, 2-7, 7-15, 15-30, 30-45, 4575, 75-105, 105-135, and 135-165 minutes. In addition, continuous HDT of 165 minutes was conducted as a control and the complete cumin seed oil was collected at the end of this time. HDT significantly affected the concentrations of the following constituents in the oil (as percentage of total oil): $\alpha$-pinene $(0.2 \%$ to $2.1 \%)$, $\beta$-pinene $(5 \%$ to $35.8 \%)$, mycrene $(0.3 \%$ to $1.7 \%)$, para-cymene $(12.0 \%$ to $26.4 \%), \gamma$-terpinene $(4.8 \%$ to $25.9 \%)$, cumin aldehyde $(3.8 \%$ to $51.1 \%)$, $\alpha$-terpinen-7-al $(0.2 \%$ to $11.2 \%)$, and $\gamma$-terpinen-7-al $(1.3 \%$ to $13.1 \%)$. Some of the constituents were eluted early in the HDP and were highest in the oil fraction collected at the beginning of the HDP, others were highest in the fractions collected midway in the HDP, and another group of constituents were eluted later and were the highest in the oil fractions collected during the last HDT (135-165 minutes). Due to their altered chemical composition, the oil fractions expressed different antioxidant capacities; the one eluted at 105-135 minutes HDT had the greatest oxygen radical absorbance capacity (ORAC) values. The ORAC values were positively correlated to the concentration of cumin aldehyde $(0.962), \alpha$-terpinene $(0.889)$ and $\gamma$-terpinene (0.717), which suggest that these compounds in cumin oil may be responsible for the measured antioxidant capacity. This study demonstrated that cumin oil with dissimilar chemical profile and antioxidant activity could be obtained from the same batch of seed by capturing oils at different timeframes during the same HDP. The resulting products (EO fractions) could have diverse industrial, medical, and environmental applications. The method for cumin seed grinding and EO extraction described in this study could be used by industry to reduce energy inputs and oil losses, and for fast oil extraction.
\end{abstract}

Received for publication 9 Mar. 2015. Accepted for publication 9 June 2015 .

This research was supported in part by Department of Plant Science funds and the Outreach School of the University of Wyoming awarded to Dr. V.D. Zheljazkov (Jeliazkov).

We thank J. Vardiman and E.A. Jeliazkova for help with the extraction and measuring of the cumin essential oil.

${ }^{1}$ Corresponding author. E-mail: valtcho.jeliazkov@, oregonstate.edu or valtcho.pubs@gmail.com.
Cumin (C. cyminum L.) is one of the important aromatic plants belonging to Apiaceae family. It has an ancient history of use as medicinal and spice plant since the Roman times (Stojanov, 1972). Cumin seed was used for the treatment of toothache, diarrhea, and epilepsy in Iranian ancient medicine (Zargari, 1989). It is also used in traditional ayurvedic medicine as a stimulant, carminative, astringent and as a remedy against indigestion, flatulence and diarrhea (Ani et al., 2006).
According to Derakhshan et al. (2008), cumin EO reduces the virulence of Klebsiella pneumoniae, one of the bacteria responsible for nosocomial infection in human. Iacobellis et al. (2005) demonstrated that EO obtained from cumin seed can control phytobacterial diseases like Clavibacter, Curtobacterium, Rhodococcus, Erwinia, Xanthomonas, and Agrobacterium. Currently, cumin is a highly valuable spice, EO crop, and medicinal plant grown from India and China, Russia, Africa, Eastern and Western Europe to America (Atanassova and Nedkov, 2004; Azeez, 2008; Deepak, 2003; Gohari and Saeidnia, 2011).

The EO of cumin seed may be extracted by steam or hydrodistillation (Atanassova and Nedkov, 2004; Beis et al., 2000; Topalov, 1962). However, since the EO is synthesized and stored inside the seed in special canals called vitae, steam distillation of the whole seeds may take several hours and may require substantial energy input (Atanassova and Nedkov, 2004). None of the literature reports had provided the optimal duration of cumin seed distillation yet. According to our experience, grinding of the cumin seed before the EO extraction may result in much faster oil extraction. However, as most EO, it doesn't dissolve in water, and evaporates quickly. Our preliminary unpublished studies ascertained that cumin seed could be ground in water and then hydrodistilled, without loss of EO. In addition, the oils distilled from ground cumin seed and captured at different timeframes, had different color and aroma. Recent reports on the effect of HDT on crushed dill (Anethum graveolens L.) (Sintim et al., 2015), coriander (Coriandrum sativum L.) (Zheljazkov et al., 2014), and fennel (Foeniculum vulgare Mill) (Burkhardt et al., 2015) demonstrated different oil constituents eluted at different distillation times. Since dill and coriander belong to the same family as cumin and have similar morphological structures (vitae) to store EO, we hypothesized that by capturing the oil fractions eluted at different times, these fractions would have dissimilar composition and possibly bioactivity, which in turn could have different research or industrial applications. These oil fractions could be exploited to produce a diversity of products.

\section{Materials and Methods}

Hydrodistillation and HDT. This experiment was carried out at the Sheridan Research and Extension Center, University of Wyoming, in 2013. In this study, we used one batch of certified bulk seed (fruits) of cumin, originated from India, and marketed by Starwest Botanicals (Rancho Cordova, CA).

The EO from all cumin seed samples was extracted via hydrodistillation in 2-L steam hydrodistillation units (Heartmagic, Rancho Santa Fe, CA). Several preliminary experiments were conducted to optimize cumin seed grinding parameters (such as duration of grinding, the amount of water needed to wash the blender, and the water to cumin seed ratio) before this hydrodistillation experiment. Consequently, in this study we used the optimized 
grinding time for cumin seed that we developed. Each sample of $200 \mathrm{~g}$ of cumin seed plus $500 \mathrm{~mL}$ of water was placed in blender, ground for $30 \mathrm{~s}$, then additional $500 \mathrm{~mL}$ of water was added to the mix, and ground for 10 more seconds. The resulting slurry was immediately transferred to the bioflask of the hydrodistillation apparatus using additional $500 \mathrm{~mL}$ of water. First distillation time started to be measured when the first drop of oil was collected by the Florentine vessel, and the entire process ended following $165 \mathrm{~min}$.

The eluted EO fractions were collected following nine HDT: $0-2,2-7,7-15,15-30,30$ 45, 45-75, 75-105, 105-135, and 135-165 min. In addition, continuous distillation (HDT of 165 min) was conducted as a control and the collected oil samples were considered as complete (full composition) cumin seed oil. The above selected HDT were based on preliminary studies conducted by the authors, and recent distillation time experiments conducted with ground seed of dill (Sintim et al., 2015), coriander (Zheljazkov et al., 2014), and fennel ( $F$. vulgare Mill) (Burkhardt et al., 2015). All the above HDTs and the control were carried out in three replicates. The resulting EO fractions and complete (full composition) cumin seed EO (control) were also analyzed in three replicates.

After all the collected oil samples were separated from water, they were weighted on analytical scale, and left in a freezer until they were analyzed.

Gas chromatography flame ionization detection essential oil quantitative analysis. All the cumin seed EO samples extracted in this study (in three replicates) were analyzed on a gas chromatograph (GC), Hewlett Packard model 6890 (Hewlett-Packard, Palo Alto, CA) with carrier gas helium at flow rate of $40 \mathrm{~cm} \cdot \mathrm{sec}^{-1}, 11.7 \mathrm{psi}\left(60{ }^{\circ} \mathrm{C}\right), 2.5 \mathrm{~mL} \cdot \mathrm{min}^{-1}$ constant flow rate, split injection $60: 1,0.5 \mu \mathrm{L}$, injector temperature $220{ }^{\circ} \mathrm{C}$. The GC oven temperature program was as follows: $60^{\circ} \mathrm{C}$ for $1 \mathrm{~min}, 10^{\circ} \mathrm{C} \cdot \mathrm{min}^{-1}$ to $250^{\circ} \mathrm{C}$ ). The column was HP-INNOWAX (cross-linked polyethylene glycol; $30 \mathrm{~m} \times 0.32 \mathrm{~mm} \times 0.5 \mu \mathrm{m}$ ), and the flame ionization detector temperature was $275{ }^{\circ} \mathrm{C}$. Individual oil components of cumin seed were expressed as percentage of the total (full composition) oil. Identification of the GC peaks representing the individual components of cumin seed oil was accomplished by internal standards (for the main oil constituents), by retention time (NIST MS library), and also using mass-spectroscopy.

Antioxidant capacity of cumin oils from different HDT. Antioxidant capacity of all cumin oil samples collected in this study (in 3 replicates +3 internal replicates $=9$ total replications per sample) was analyzed using the ORAC $_{\text {oil }}$ method (Huang et al., 2002a, 2002b). Specific details regarding this method were previously described by Zheljazkov et al. (2013c). The antioxidant capacity of the cumin oil samples was measured using Trolox as a standard (6-hydroxy-2,5,7,8-tetramethylchroman2-carboxylic acid), the results are expressed as micromoles Trolox per gram.

Statistical methods. The effect of distillation time on oil content (\%), and the concentration ( $\%$ ) of $\alpha$-pinene, $\beta$-pinene, myrcene, para-cymene, $\gamma$-terpinene, cumin aldehyde, $\alpha$-terpinen-7-al, $\gamma$-terpinen-7-al, and ORAC was determined using a one-way analysis of variance. For each response, the validity of model assumptions (normal distribution and constant variance of the error terms) was verified by examining the residuals as described in Montgomery (2013). Normal probability plot of the residuals and the Anderson-Darling test for normality were used to verify the normal distribution assumption, and plot of the residuals vs. fitted values was used to verify the constant variance assumption. Randomization ensured the validity of the independence assumption. Since the effect of distillation time was significant $(P$ value $<0.05)$ on all responses, multiple means comparison was completed using Duncan's multiple range test at the $5 \%$ level of significance to compare the nine HDT treatments; and to compare the control treatment with the other HDT treatments, Dunnett's test at the 5\% level of significance was used. The analysis was completed using the General Linear Model (GLM) procedure of SAS (SAS Institute Inc., 2010).

The relationships between HDT and oil content (\%), and between HDT and the concentration of $\alpha$-pinene, $\beta$-pinene, myrcene, and $\gamma$-terpinene were adequately modeled by the Power model (Eq. [1]); the relationships between HDT and the concentration of cumin aldehyde, $\alpha$-terpinen-7-al, and ORAC were adequately modeled by the Michaelis-Menten model (Eq. [2]); and the relationships between HDT and the concentrations of para-cymene, and $\gamma$-terpinen7-al were adequately described by the third order polynomial model (Eq. [3]). The parameters of the nonlinear models (Michaelis-Menten and Power) were estimated iteratively using the Nonlinear (NLIN) procedure of SAS (SAS Institute Inc., 2010) and the fitted models met all adequacy requirements (Bates and Watts, 2007).

$$
\begin{gathered}
Y=\theta_{1} X^{\theta_{2}}+\varepsilon \\
Y=\frac{\theta_{1} X}{\theta_{2}+X}+\varepsilon \\
Y=\beta_{0}+\beta_{1} X+\beta_{2} X^{2}+\beta_{3} X^{3}+\varepsilon
\end{gathered}
$$

where $Y$ is the dependent (response) variable, $X$ is the independent (HDT) variable, and the error term $\epsilon$ is assumed to have normal distribution with constant variance. Validity of the normality, constant variance, and independence assumptions on the error terms was verified by examining the residuals (Bates and Watts, 2007).

\section{Results}

The effect of HDT was significant $(P$ value $<$ $0.05)$ on all responses. The content of captured EO fractions fluctuated markedly during the HDP. The greatest amount of oil $(1.32 \%$, or gram oil $/ 100$ seed) was eluted early in the HD process, at $0-2$ min HDT, whereas the lowest $(0.090 \%)$ was eluted at 135-165-min HDT (Table 1; Fig. 1). Generally, the yields of the oil fractions decreased with each subsequent collection after the 0-2 min HDT (Fig. 1).

In comparison with the total oil content, the concentration of individual oil constituents (out of the total oil) showed diverse trends. The concentration of various constituents in total oil ranged within the following limits: $\alpha$-pinene $2.12 \%$ to $0.18 \%, \beta$-pinene $35.8 \%$ to $5.0 \%$, and myrcene $1.67 \%$ to $0.26 \%$; these were highest in the oil fraction collected during the 0-2 min HDT and decreased with the subsequent collections until 75-105 min HDT when they reached their lowest respective concentrations (Table 1; Fig. 1). The concentration of para-cymene (26.4\% to $12.0 \%$ concentration range in the total oil) was also highest in the oil fraction eluted at $0-2 \mathrm{~min}$ HDT and the lowest at the HDT between 30 and $135 \mathrm{~min}$ (Table 1; Fig. 1)

The concentration of $\gamma$-terpinene $(25.9 \%$ to $4.8 \%$ concentration range of total oil) was also the highest in the oil fraction collected at 0-2 min HDT and gradually decreased with the increase of HDT, to reach the lowest value in the oil fraction eluted at 105-135 and 135-165 min HDT (Table 2; Fig. 1). The concentrations of cumin aldehyde, the major oil constituent $(3.8 \%$ to $51.1 \%$ of the total oil), and $\alpha$-terpinen- 7 -al $(0.18 \%$ to $11.17 \%)$ were the lowest in the oil fraction collected at 0-2-min and reached their respective maximums in the one collected at 75-105 and

Table 1. Mean oil content ( $\%$ oil of seed weight) and the concentration ( $\%$ constituent out of the total oil) of $\alpha$-pinene, $\beta$-pinene, myrcene, and para-cymene obtained from the nine hydrodistillation times (HDT) and control (full oil composition with continuous 165 min HDT).

\begin{tabular}{lclccc}
\hline HDT $(\mathrm{min})$ & Oil content & $\alpha$-pinene & $\beta$-pinene & Myrcene & Para-cymene \\
\hline $0-2$ & $1.32 \mathrm{a}^{\mathrm{z}}$ & $2.12 \mathrm{a}$ & $35.8 \mathrm{a}$ & $1.67 \mathrm{a}$ & $26.4 \mathrm{a}$ \\
$2-7$ & $0.83 \mathrm{~b}$ & $1.12 \mathrm{~b}$ & $21.2 \mathrm{~b}$ & $1.11 \mathrm{~b}$ & $21.9 \mathrm{~b}$ \\
$7-15$ & $0.58 \mathrm{c}$ & $1.01 \mathrm{~b}^{* y}$ & $17.3 \mathrm{c} *$ & $0.88 \mathrm{c}^{*}$ & $17.3 \mathrm{c}^{*}$ \\
$15-30$ & $0.62 \mathrm{c}$ & $0.73 \mathrm{c}$ & $12.7 \mathrm{~d}$ & $0.62 \mathrm{~d}$ & $14.0 \mathrm{~d}^{*}$ \\
$30-45$ & $0.44 \mathrm{~cd}$ & $0.53 \mathrm{~d}$ & $10.0 \mathrm{e}$ & $0.47 \mathrm{e}$ & $12.6 \mathrm{de}$ \\
$45-75$ & $0.60 \mathrm{c}$ & $0.44 \mathrm{~d}$ & $8.8 \mathrm{e}$ & $0.42 \mathrm{e}$ & $12.1 \mathrm{e}$ \\
$75-105$ & $0.29 \mathrm{de}$ & $0.21 \mathrm{e}$ & $5.5 \mathrm{f}$ & $0.31 \mathrm{f}$ & $12.0 \mathrm{e}$ \\
$105-135$ & $0.19 \mathrm{ef}$ & $0.18 \mathrm{e}$ & $5.0 \mathrm{f}$ & $0.27 \mathrm{f}$ & $13.7 \mathrm{de}^{*}$ \\
$135-165$ & $0.09 \mathrm{f}$ & $0.21 \mathrm{e}$ & $5.8 \mathrm{f}$ & $0.26 \mathrm{f}$ & $16.6 \mathrm{c}^{*}$ \\
Control & $4.74^{\mathrm{x}}$ & 0.96 & 18.0 & 0.93 & 16.3 \\
\hline
\end{tabular}

${ }^{\mathrm{z}}$ Within each column, means of the nine HDTs (other than the control) sharing the same letter are not significantly different according to Duncan's multiple range test at the 5\% level.

${ }^{y}$ Within each concentration, means with * are not significantly different from the control treatment according to Dunnett's test at the 5\% level.

${ }^{\mathrm{x}}$ The mean oil content for control is not compared with the other means. 

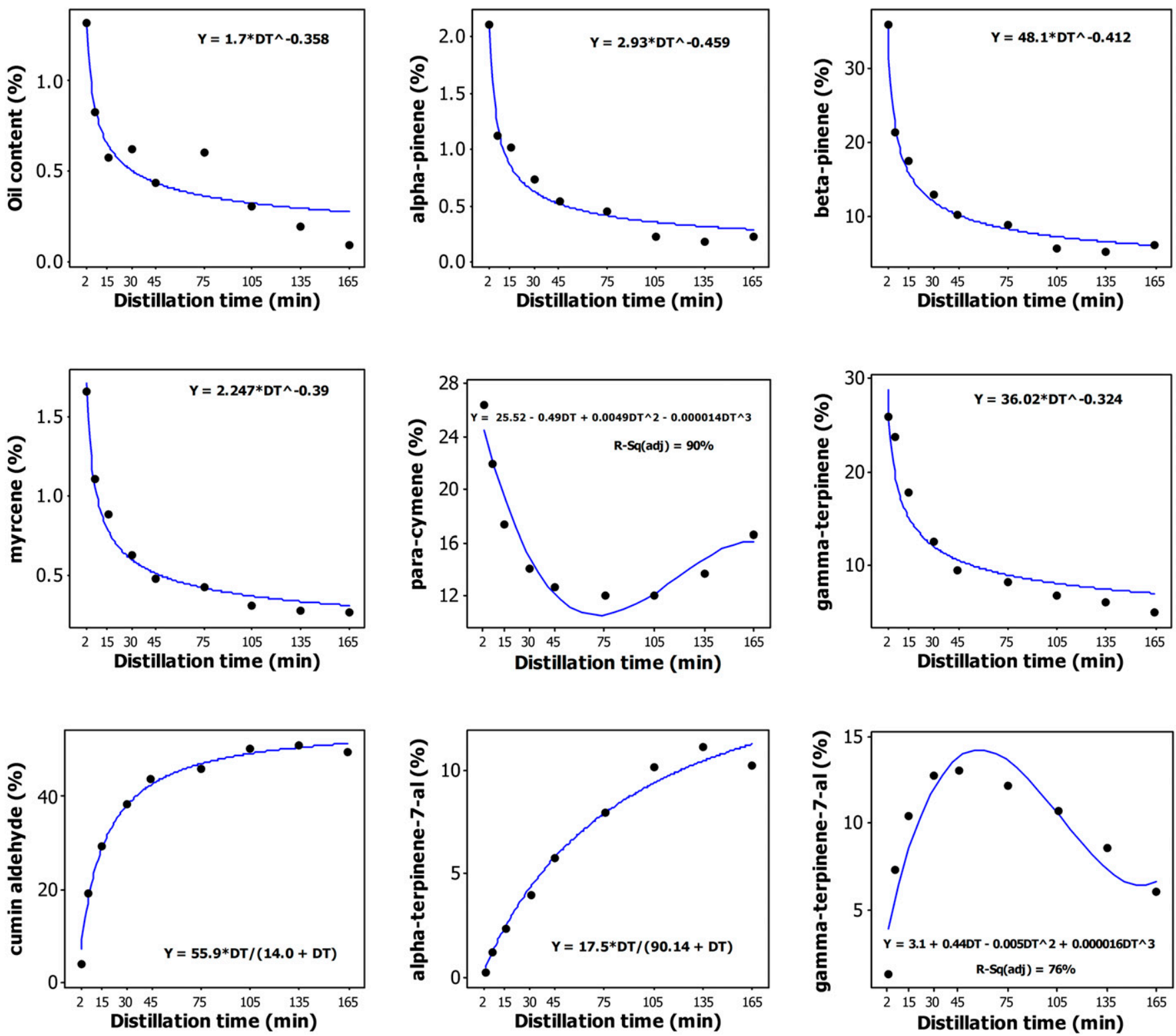

Fig. 1. Plot of distillation time vs. oil content ( $\%$ oil out of the seed weight), and the concentration ( $\%$ oil constituent out of the total oil) of $\alpha$-pinene, $\beta$-pinene, myrcene, para-cymene, $\gamma$-terpinene, cumin aldehyde, $\alpha$-terpinen-7-al, and $\gamma$-terpinen-7-al along with the fitted (solid line) power, Michaelis-Menten, and third order polynomial models.

105-135 min (Table 2; Fig. 1). The concentration of $\gamma$-terpinen-7-al $(1.3 \%$ to $13.2 \%)$ was also the lowest in the oil fraction at 0 2 min and highest in the one collected at 1575 min HDT (Table 2; Fig. 1). The composition of cumin seed oil collected in the $0-165 \mathrm{~min}$ nonstop process (the control) had significantly different chemical profile from the compositional profiles of oil fractions eluted at different times (Tables 1 and 2).

The observed antioxidant capacities differed between the oil fractions; generally the antioxidant capacity of the oil fraction collected at 0-2 min HDT was low, and increased with each subsequent collection to reach maximum values with the fraction collected at 105-135 min (Table 2; Fig. 2). The antioxidant capacity of the control (complete EO) was similar to the oil fraction collected at $30 \mathrm{~min}$. The uninterrupted $165 \mathrm{~min}$ distillation gave significantly lower ORAC value than the fraction collected at $135 \mathrm{~min}$, and similar to the ORAC of the fraction collected at 1530 min HDT (Table 2). The regression model (that does not include the control) showed the potentially maximum estimated value of ORAC to be 383.8 (Fig. 2). The ORAC values were positively correlated to the concentration of cumin aldehyde (0.962), $\alpha$-terpinene $(0.889)$ and $\gamma$-terpinene $(0.717)$ and negatively correlated to the concentrations of $\alpha$-pinene, $\beta$-pinene, myrcene, para-cymene, and $\gamma$-terpinene (Fig. 2).

\section{Discussion}

Due to grinding of the cumin seeds before hydrodistillation procedure, the majority of the EO eluted very early. This rapid oil extraction leads to significant time and energy savings in the steam distillation or hydrodistillation of cumin seed. Beis et al.
(2000) and Ghafari et al. (2014) had to distill for $3 \mathrm{~h}$ to completely extract $\mathrm{EO}$ of cumin. Both researchers had used whole cumin seed.

Different oil fractions significantly differed in chemical composition $(P$ value $<$ $0.05)$, hence confirming the hypothesis of the study. Generally, the oil content (yield of oil per $100 \mathrm{~g}$ seed) and the concentration of $\alpha$-pinene, $\beta$-pinene, mycrene, para-cymene, and $\gamma$-terpinene were highest in the oil fractions collected at the initial HDT, whereas the concentration of cumin aldehyde, $\alpha$-terpinen7 -al and $\gamma$-terpinen-7-al was lowest at the same fractions. The overall oil content in the control (complete cumin seed oil) was similar to that of the previously reported ones (Bettaieb et al., 2011; Jirovetz et al., 2005; Sowbhagya et al., 2008). However, Sowbhagya et al. (2008) and Bettaieb et al. (2011) distilled for $3 \mathrm{~h}$ and $2 \mathrm{~h}$, respectively, and their cumin seed samples originated from different geographic regions. 
Table 2. Mean concentration (\% constituent out of the total oil) of $\gamma$-terpinene, cumin aldehyde, $\alpha$-terpinen-7-al, $\gamma$-terpinen-7-al, and oxygen radical absorbance capacity (ORAC) ( $\mu \mathrm{m}$ Trolox equivalent per gram oil) obtained from the nine hydrodistillation times (HDT) and control (straight nonstop HDT of $165 \mathrm{~min})$.

\begin{tabular}{lccccc}
\hline HDT $(\mathrm{min})$ & $\gamma$-terpinene & Cumin aldehyde & $\alpha$-terpinen-7-al & $\gamma$-terpinen-7-al & ORAC \\
\hline $0-2$ & $25.9 \mathrm{a}^{\mathrm{z}}$ & $3.8 \mathrm{f}$ & $0.18 \mathrm{~g}$ & $1.27 \mathrm{f}$ & $25 \mathrm{f}$ \\
$2-7$ & $23.8 \mathrm{~b}$ & $19.0 \mathrm{e}$ & $1.13 \mathrm{~g}$ & $7.27 \mathrm{~d}$ & $108 \mathrm{e}$ \\
$7-15$ & $17.7 \mathrm{c}^{* \mathrm{y}}$ & $29.2 \mathrm{~d}^{*}$ & $2.26 \mathrm{f}$ & $10.4 \mathrm{~b}^{*}$ & $182 \mathrm{~d}^{*}$ \\
$15-30$ & $12.6 \mathrm{~d}$ & $38.4 \mathrm{c}$ & $3.96 \mathrm{e}^{*}$ & $12.7 \mathrm{a}$ & $257 \mathrm{c}^{*}$ \\
$30-45$ & $9.3 \mathrm{e}$ & $43.9 \mathrm{~b}$ & $5.77 \mathrm{~d}^{*}$ & $13.1 \mathrm{a}$ & $302 \mathrm{~b}$ \\
$45-75$ & $8.2 \mathrm{f}$ & $46.0 \mathrm{~b}$ & $7.9 \mathrm{c}$ & $12.2 \mathrm{a}$ & $285 \mathrm{bc}$ \\
$75-105$ & $6.7 \mathrm{~g}$ & $50.5 \mathrm{a}$ & $10.2 \mathrm{~b}$ & $10.7 \mathrm{~b}^{*}$ & $276 \mathrm{bc}$ \\
$105-135$ & $5.9 \mathrm{gh}$ & $51.1 \mathrm{a}$ & $11.2 \mathrm{a}$ & $8.5 \mathrm{c}^{*}$ & $394 \mathrm{a}$ \\
$135-165$ & $4.8 \mathrm{~h}$ & $49.8 \mathrm{a}$ & $10.2 \mathrm{ab}$ & $6.0 \mathrm{e}$ & NA $^{\mathrm{x}}$ \\
Control & 17.6 & 27.8 & 4.62 & 9.6 & 219 \\
\hline
\end{tabular}

${ }^{\mathrm{z}}$ Within each column, means of the nine HDTs (other than the control) sharing the same letter are not significantly different according to Duncan's multiple range test at the $5 \%$ level.

${ }^{\mathrm{y}}$ Within each concentration, means with * are not significantly different from the control treatment according to Dunnett's test at the 5\% level.

${ }^{\mathrm{x}} \mathrm{NA}=$ Not available.

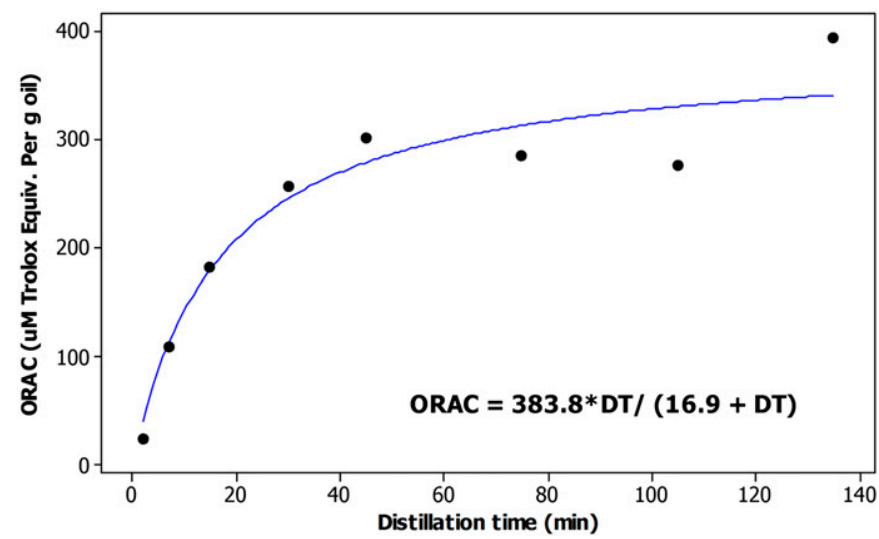

Fig. 2. Plot of distillation time vs. oxygen radical absorbance capacity (ORAC) ( $\mu$ mol Trolox equivalents per gram oil) along with the fitted (solid line) Michaelis-Menten model.

The oil from the control (full oil compositioncontinuous nonstop $165 \mathrm{~min}$ HDP) in our study was close to the oil content reported by Sowbhagya et al. (2008) and Bettaieb et al. (2011). On the other hand, Jirovetz et al. (2005) extracted oil from 36 years preserved cumin seed and found surprisingly higher average oil content (5.4\%), most probably due to substantial water loss during such a long storage. However, it is difficult to cause variations in oil yield since it can be attributed to many reasons such as genetic factors, maturity stage, environmental factors and distillation times (Lawrence, 2002; Zheljazkov et al., $2013 \mathrm{c}$ ), grinding method, and how finely the seed was ground before distillation (Nadeem and Riaz, 2002).

The major components of cumin seed oil in our study were cumin aldehyde, $\beta$-pinene, para-cymene, $\gamma$-terpinene, $\gamma$-terpinen- 7 -al, and $\alpha$-terpinen-7-al, and it is in agreement with previous reports on cumin seed oil composition (Bettaieb et al., 2011; Jirovetz et al., 2005; Yang et al., 2012).

The results from this study demonstrated that higher contents of the most important cumin seed oil constituents can be captured in the first 2 min of distillation, except for the cumin aldehyde, which was highest in the oil fraction eluted at 105-135-min HDT. The concentrations of the major oil constituents of cumin seed oil obtained at that elution time were much higher than the previously reported cumin oil composition (Bettaieb et al., 2011; Ghafari et al., 2014; Topalov, 1962). Hence, we obtained cumin seed oil fractions with unique compositions. Furthermore, most of the researchers used much longer distillation times to achieve complete oil extraction (Ghafari et al., 2014). Thus, our study suggests that more efficient cumin seed oil extraction can be achieved, if we understand the cumin ground seed hydrodistillation extraction kinetics.

The oil fractions eluted at different times showed distinct antioxidant capacity, opening the possibility for using this method to obtain cumin seed oil with differential antioxidant capacity. The oil fractions eluted late were higher in antioxidant capacity than the oil fractions eluted early. This can be accounted for the higher concentration of cumin aldehyde, $\alpha$-terpinene -7-al, and $\gamma$-terpinene-7-al found in late distillate fractions. These compounds inhibit lipid peroxidation and enhance the activity of endogenous antioxidant enzymes (Maestri et al., 2006). Higher boiling point of cumin aldehyde, $\alpha$-terpinene7 -al, and $\gamma$-terpinene-7-al than other constituents of cumin EO may be the reason behind late elution of these chemicals. Recent research with other crops, such as dill seed (A. graveolens L.) (Sintim et al., 2015), male and female rocky mountain juniper (Juniperus scopulorum Sarg.) (Zheljazkov et al., 2012; Zheljazkov et al., 2013b), sweet wormwood (Artemisia annua L.) (Zheljazkov et al., 2013a), fennel ( $F$. vulgare Mill.) (Zheljazkov et al., 2013c), coriander (C. sativum L.) (Zheljazkov et al., 2014), and fennel (F. vulgare Mill) (Burkhardt et al., 2015), also found that distillation time can be used to obtain oil fractions with different antioxidant capacity from these plant species.

The correlation coefficients between the concentration of individual oil constituents in the oil and the ORAC values suggest that increasing concentrations of cumin aldehyde, $\alpha$-terpinene, and $\gamma$-terpinene may contribute to increase in antioxidant capacity. This finding is somewhat in line with a recent study by Chen et al. (2014), who found that $\gamma$-terpinene was the most efficient antioxidant compound in cumin oil, though the authors used a different method 2.2Diphenyl-1-Picrylhydrazyl (DPPH) for measuring the antioxidant capacity. There are some reports on the antioxidant capacity of cumin seed oil (Einafshar et al., 2012; Gachkar et al., 2007), however, these were determined using DPPH radical-scavenging capacity, which makes comparison difficult. As the ORAC values in our study were positively correlated to the concentration of cumin aldehyde (0.962), $\alpha$-terpinene (0.889), and $\gamma$-terpinene $(0.717)$, we assume these compounds in cumin oil are mostly responsible for the measured antioxidant capacity of the oil. The United States Department of Agriculture has recently concluded that ORAC values for foods are not nutritionally relevant. However, ORAC assay is still used to measure the usefulness of antioxidants in preservation of drugs. Therefore, it seems the oil fraction obtained late in the HDP could have a potential as a preservative for organic nutritional supplements. This is the first study to demonstrate that various cumin oil fractions, due to their different chemical composition possess different antioxidant capacities.

\section{Conclusion}

A method for grinding of cumin seed in water was developed and optimized for decreasing the time and the energy input for hydrodistillation of cumin seed. The method used in this study was designed to produce cumin oil fractions with diverse chemical profile and antioxidant activity by capturing the eluted oil fractions at different time points.

The cumin oil fractions collected at nine HDT frames exhibited specific chemical profile and antioxidant activity. Some oil constituents reached their maximum in the oil fraction eluted at the initial HDT (0-2 min), others, later in the HDT process.

The positive correlations between the concentration of individual oil constituents in the oil and the ORAC values suggest that increasing concentrations of cumin aldehyde, 
$\alpha$-terpinene, and $\gamma$-terpinene may contribute to increased antioxidant capacity. This study demonstrated that cumin oil fractions with different chemical profile and bioactivity could be obtained from the same batch of seed. Since the most important cumin oil fractions responsible for distinct fragrance can be collected early and the latter fractions collected have the greatest antioxidant activity, our findings could be exploited to produce a diversity of products; early distillate fractions could be used as flavoring agent, whereas late distillate fractions could be used as food and drug preservatives. The findings from this study could be used by industry and researchers for quick extraction of cumin seed EO fractions with different composition and bioactivity from the same batch of cumin seed.

\section{Literature Cited}

Ani, V., M.C. Varadaraj, and K.A. Naidu. 2006. Antioxidant and antibacterial activities of polyphenolic compounds from bitter cumin (Cuminum nigrum L.). Eur. Food Res. Technol. 224:109-115.

Atanassova, M. and N. Nedkov. 2004. Essential oil and medicinal crops. Kameja Press, Sofia, Bulgaria.

Azeez, S. 2008. Cumin, p. 211-226. In: V.A. Parthasarathy, B. Chempakam, and T.J. Zachariah (eds.). Chemistry of spices, $\mathrm{CAB}$ International, Wallingford, Oxfordshire, UK.

Bates, D.M. and D.G. Watts. 2007. Nonlinear regression and its applications, p. 90-96. Wiley, New York, NY.

Beis, S.H., N. Azcan, T. Ozek, M. Kara, and K.H.C. Baser. 2000. Production of essential oil from cumin seeds. Chem. Nat. Compd. 36:265-268.

Bettaieb, I., S. Bourgou, J. Sriti, K. Msaada, F. Limam, and B. Marzouk. 2011. Essential oils and fatty acids composition of Tunisian and Indian cumin (Cuminum cyminum L.) seeds: A comparative study. J. Sci. Food Agr. 91:2100-2107.

Burkhardt, A., H.Y. Sintim, A. Gawde, C.L. Cantrell, T. Astatkie, V.D. Zheljazkov, and V. Schlegel. 2015. Method for attaining fennel (Foeniculum vulgare Mill.) seed oil fractions with different composition and antioxidant capacity. J. Appl. Res. Med. Arom. Plants. doi:10.1016/j.jarmap.2015.04.003.

Chen, Q.Q., Z.L. Gan, J.H. Zhao, Y. Wang, S.M. Zhang, J.M. Li, and Y.Y. Ni. 2014. In vitro comparison of antioxidant capacity of cumin (Cuminum cyminum L.) oils and their main components. LWT-Food Sci. Technol. (Campinas.) 55:632-637.

Deepak. 2003. Importance of Cuminum cyminum L. and Carum carvi L. in traditional medicaments: A review. Ind. J. Tradit. Knowl. 12:300-307.
Derakhshan, S., M. Sattari, and M. Bigdeli. 2008. Effect of subinhibitory concentrations of cumin (Cuminum cyminum L.) seed essential oil and alcoholic extract on the morphology, capsule expression and urease activity of Klebsiella pneumoniae. Intl. J. Antimicrob. Agents 32:432-436.

Einafshar, S., H. Poorazrang, R. Farhoosh, and S.M. Seiedi. 2012. Antioxidant activity of the essential oil and methanolic extract of cumin seed (Cuminum cyminum). Eur. J. Lipid Sci. Technol. 114:168-174.

Gachkar, L., D. Yadegari, M.B. Rezaei, M. Taghizadeh, S.A. Astaneh, and I. Rasooli. 2007. Chemical and biological characteristics of Cuminum cyminum and Rosmarinus officinalis essential oils. Food Chem. 102:898-904.

Ghafari, Z., A. Alizadeh, and R.B. Samani. 2014. Antimicrobial activity and essential oil composition of Cuminum cyminum L. and Carum carvi L. seeds from Iran. Intl. J. Biosci. 6:153159.

Gohari, A.R. and S.A. Saeidnia. 2011. A review on phytochemistry of Cuminum cyminum seeds and its standards from field to market. Pharm. J. 3:1-5.

Huang, D., B. Ou, M. Hampsch-Woodill, J. Flanagan, and E.K. Demmer. 2002a. Development and validation of oxygen radical absorbance capacity assay for lipophilic antioxidants using randomly methylate B-cylodextrin as the solubility enhancer. J. Agr. Food Chem. 50:1815-1821.

Huang, D., B. Ou, M. Hampsch-Woodill, J. Flanagan, and R. Prior. 2002b. High-throughput assay of oxygen radical absorbance capacity (ORAC) using a multichannel liquid handling system coupled with a microplate fluorescence reader in 96-well format. J. Agr. Food Chem. 50:44374444.

Iacobellis, N.S., P.L. Cantore, F. Capasso, and F. Senatore. 2005. Antibacterial activity of Cuminum cyminum L. and Carum carvi L. Essential Oils J. Agr. Food Chem. 53:57-61.

Jirovetz, L., G. Buchbauer, A.S. Stoyanova, E.V. Georgiev, and S.T. Damianova. 2005. Composition quality control and antimicrobial activity of the essential oil of cumin (Cuminum cyminum L.) seeds from Bulgaria that had been stored for up to 36 years. Intl. J. Food Sci. Technol. 40:305-310.

Lawrence, M.B. 2002. From the sensation to the synthesis in advances in flavours and fragrances, p. 57-83. In: K. Swift (ed.). Special publications (277), Cambridge: Royal Society of Chemistry, Cambridge, MA.

Maestri, D.M., V. Nepote, A.L. Lamarque, and J.A. Zygadlo. 2006. Natural products as antioxidants, p. 105-135. In: F. Imperato (ed.). Phytochemistry: Advances in research. Research Signpost, Cordoba, Argentina.
Montgomery, D.C. 2013. Design and analysis of experiments, p. 80-88. 8th ed. Wiley, New York, NY.

Nadeem, M. and A. Riaz. 2002. Cumin (Cuminum cyminum) as a potential source of antioxidants. Pak. J. Food Sci. 22:101-107.

SAS Institute Inc. 2010. SAS/STAT ${ }^{\circledR} 9.3$ user's guide. SAS Institute Inc., Cary, NC.

Sintim, H.Y., A. Burkhardt, A. Gawde, C.L. Cantrell, T. Astatkie, A. Obour, V.D. Zheljazkov, and V. Schlegel. 2015. Hydrodistillation time affects dill seed essential oil yield, composition, and bioactivity. Ind. Crops Prod. 63:190-196.

Sowbhagya, H.B., B.V. Sathyendra Rao, and N. Krishnamurthy. 2008. Evaluation of size reduction and expansion on yield and quality of cumin (Cuminum cyminum) seed oil. J. Food Eng. 84:595-600.

Stojanov, N. 1972. Cumin, p. 266-267. In: N. Stojanov (ed.). Our medicinal plants. Nauka and Iskustvo Press, Sofia, Bulgaria.

Topalov, V.D. 1962. Essential oil and medicinal plants, p. 246-251. Hr. G. Danov Press, Plovdiv, Bulgaria.

Yang, J., W. Hongmin, C. Yu, Y. Shi, and H. Zhang. 2012. Extraction of the volatile and semivolatile compounds in seeds of Cuminum cyminum L. using hydrodistillation followed by headspace-Ionic liquid-based single-drop microextraction. Chromatographia 75:14351443.

Zheljazkov, V.D., T. Astatkie, and V. Schlegel. 2014. Hydrodistillation extraction time effect on essential oil yield, composition, and bioactivity of coriander oil. J. Oleo Sci. 60:335342.

Zheljazkov, V.D., T. Astatkie, E.A. Jeliazkova, and V. Schlegel. 2012. Distillation time alters essential oil yield, composition, and antioxidant activity of male Juniperus scopulorum trees. J. Oleo Sci. 61:537-546.

Zheljazkov, V.D., T. Astatkie, T. Horgan, V. Schlegel, and X. Simonnet. 2013a. Distillation time effect on essential oil yield, composition, and antioxidant capacity of sweet sagewort (Artemisia annua L.) oil. HortScience 48:1288-1292.

Zheljazkov, V.D., T. Astatkie, E.A. Jeliazkova, A.O. Tatman, and V. Schlegel. 2013b. Distillation time alters essential oil yield, composition and antioxidant capacity of female Juniperus scopulorum trees. J. Essent. Oil Res. 25:62-69.

Zheljazkov, V.D., T. Horgan, T. Astatkie, and V. Schlegel. 2013c. Distillation time modifies essential oil yield, composition, and antioxidant capacity of fennel (Foeniculum vulgare Mill). J. Oleo Sci. 62:665-672.

Zargari, A. 1989. Medicinal plants, p. 519-521. Tehran University Press, Tehran. 